\title{
Nuclear import of the Drosophila Rel protein Dorsal is regulated by phosphorylation
}

\author{
Eric A. Drier, ${ }^{1-3}$ Leslie H. Huang, ${ }^{2}$ and Ruth Steward ${ }^{2,4}$ \\ ${ }^{1}$ Department of Molecular Biology, Princeton University, Princeton, New Jersey 08544 USA; ${ }^{2}$ The Waksman Institute, \\ Department of Molecular Biology and Biochemistry, Rutgers University, Piscataway, New Jersey 08854-8020 USA
}

In Drosophila, dorsal-ventral polarity is determined by a maternally encoded signal transduction pathway that culminates in the graded nuclear localization of the Rel protein, Dorsal. Dorsal is retained in the cytoplasm by the IкB protein, Cactus. Signal-dependent phosphorylation of Cactus results in the degradation of Cactus and the nuclear targeting of Dorsal. We present an in-depth study of the functional importance of Dorsal phosphorylation. We find that Dorsal is phosphorylated by the ventral signal while associated with Cactus, and that Dorsal phosphorylation is essential for its nuclear import. In vivo phospholabeling of Dorsal is limited to serine residues in both ovaries and early embryos. A protein bearing mutations in six conserved serines abolishes Dorsal activity, is constitutively cytoplasmic, and appears to eliminate Dorsal phosphorylation, but still interacts with Cactus. Two individual serine-to-alanine mutations produce unexpected results. In a wild-type signaling background, a mutation in the highly conserved PKA site (S312) produces only a weak loss-of-function; however, it completely destabilizes the protein in a cactus mutant background. Significantly, the phosphorylation of another completely conserved serine (S317) regulates the high level of nuclear import found in ventral cells. We conclude that the formation of a wild-type Dorsal nuclear gradient requires the phosphorylation of both Cactus and Dorsal. The strong conservation of the serines suggests that phosphorylation of other Rel proteins is essential for their proper nuclear targeting.

[Key Words: Rel family; dorsal-ventral polarity; phosphorylation; nuclear import; signal transduction]

Received September 17, 1998; revised version accepted January 13, 1999.

Members of the Rel family of transcription factors participate in a wide array of biological processes including vertebrate antibody synthesis, innate immunity, inflammatory responses, and limb development (Siebenlist et al. 1994; Verma et al. 1995; Bushdid et al. 1998; Kanegae et al. 1998), as well as Drosophila immunity and dorsalventral patterning (for review, see Drier and Steward 1997). Rel proteins share a highly related 300-amino-acid amino-terminal Rel homology region (RHR). They are retained in the cytoplasm through direct interaction with IкB proteins (Sen and Baltimore 1986; Baeuerle and Baltimore 1988; for review, see Whiteside and Isreal 1997). In response to activating signals, Rel proteins are released from this interaction, enabling their translocation to the nucleus.

Systematic genetic screens have identified 12 maternal effect genes required to establish the dorsal-ventral axis of Drosophila embryos (Anderson and Nüsslein-Volhard 1984; Schüpbach and Wieschaus 1989|. Molecular

\footnotetext{
${ }^{3}$ Present address: Cold Spring Harbor Laboratory, Cold Spring Harbor, New York 11724 USA.

${ }^{4}$ Corresponding author.

E-MAIL steward@MBCL.rutgers.edu; FAX (732) 445-5735.
}

analyses and genetic epistasis experiments have established that the products of these genes comprise a signaltransduction pathway whose end result is the formation of a ventral-to-dorsal nuclear gradient of the Rel protein, Dorsal (Steward 1989; for review, see Morisato and Anderson 1995; Drier and Steward 1997). Ten of the identified genes encode products that positively regulate the nuclear import of Dorsal (Roth et al. 1989; Rushlow et al. 1989; Steward 1989). The product of the last gene, cactus, is an IкB protein that interacts with Dorsal to retain Dorsal in the cytoplasm (Roth et al. 1991; Geisler et al. 1992; Kidd 1992).

Beside Cactus and Dorsal, two other components of the intracellular dorsal-ventral pathway are homologous to proteins implicated in vertebrate Rel systems. Toll, a transmembrane protein homologous to hToll and the IL-1 receptor cytoplasmic domain, functions to receive and transduce an extracellular signal (Hashimoto et al. 1988; Schneider et al. 1991; Medzhitov et al. 1997). The products of the tube and pelle genes relay this signal from Toll to the cytoplasmic Dorsal-Cactus complex (Hecht and Anderson 1993). The Tube protein is novel, but Pelle is a serine/threonine kinase homologous to IRAK, a kinase found to rapidly associate with the intra- 
cellular domain of the IL-1 receptor upon ligand binding (Letsou et al. 1991; Shelton and Wasserman 1993; Cao et al. 1996).

Cactus activity is regulated by both signal-independent and signal-dependent processes that target it for degradation (Belvin et al. 1995). In dorsal protein-null backgrounds, no Cactus is present as a result of the signal-independent process, which acts to degrade free Cactus and achieve a dynamic equilibrium between Dorsal and Cactus (Govind et al. 1993). The signal-dependent degradation of Cactus involves serine phosphorylation events in the amino-terminal region analogous to those controlling IкB- $\alpha$ (Brown et al. 1995; Reach et al. 1996). Thus, the dorsal-ventral signal transduction pathway functions, at least in part, to regulate the degradation of Cactus. However, a weak nuclear gradient of Dorsal exists in the complete absence of Cactus activity, indicating that the signal also acts directly through Dorsal to regulate its nuclear uptake (Bergmann et al. 1996).

In addition to the IкB modification, RelA (p65), c-Rel, and Dorsal are also phosphorylated in response to signaling (Whalen and Steward 1993; Gillespie and Wasserman 1994; Li et al. 1994; Naumann and Scheidereit 1994). Constitutively active alleles of Toll, and loss-of-function cactus alleles, cause Dorsal to translocate to all nuclei along the dorsal-ventral axis (Roth et al. 1989; Steward 1989). A hyperphosphorylated form of Dorsal predominates in both of these genetic backgrounds. When the signal transduction pathway is disrupted, Dorsal remains cytoplasmic, and the hyperphosphorylated form of Dorsal is absent (Whalen et al. 1993; Gillespie et al. 1994).

Genetic experiments indicate that Dorsal nuclear translocation is regulated on two levels (Roth et al. 1991; Govind et al. 1993). Both require the dissociation of the Dorsal-Cactus complex. Free Dorsal achieves the first, basal level of nuclear translocation normally attained in lateral cells. This import does not require the signal because it is observed in embryos from mothers simultaneously mutant for the signal as well as cactus. The second level of nuclear translocation is signal dependent, and requires additional events to achieve the higher concentrations of nuclear Dorsal normally seen in ventral cells.

We studied the role of Dorsal phosphorylation in the regulation of its activity by determining on which amino acid Dorsal is phosphorylated, and complemented this work by in vivo functional analysis of mutations in potentially phosphorylated amino acids. We find that Dorsal is subject to signal-dependent phosphorylation while associated with Cactus in the cytoplasm. In vivo labeling indicates that Dorsal phosphorylation is restricted to serine residues in both ovaries and early embryos. We made serine-to-alanine mutations in conserved serines within the RHR of Dorsal and measured their in vivo effects using transgenic flies. The transgenes were controlled by a dorsal genomic promoter fragment that conferred lineindependent expression identical to endogenous dorsal alleles. A mutant simultaneously altering six highly conserved serines within the RHR (6XS-A-Dorsal) abolishes
Dorsal activity, resulting in completely dorsalized embryos. This mutant protein is stable, constitutively cytoplasmic, and it eliminates all measurable Dorsal phosphorylation; however, it retains its ability to bind Cactus normally and, thus, is not severely misfolded. These results indicate that Dorsal phosphorylation is essential for its nuclear localization.

When each of the serines is changed individually to alanine, we find that one mutation (S79A), that disrupts a predicted casein kinase II site, does not affect the function of Dorsal, whereas the remaining five serine-to-alanine mutants measurably alter Dorsal activity. Three (S70A, S103A, and S213A) have indistinguishable weak loss-of-function effects. A mutation in the highly conserved and well-studied putative protein kinase A (PKA) site (S312A) shows a similar weak loss-of-function phenotype that is temperature sensitive. In contrast to all other serine-to-alanine mutations, this mutant protein is destabilized in the absence of Cactus.

Most importantly, we find that a mutation of S317A significantly reduces the function of Dorsal, as measured by both embryonic phenotype and nuclear localization. The hyperphosphorylated form of Dorsal is also missing in this mutant. Taken together, these data indicate that S317 is the target of the signal-dependent phosphorylation of Dorsal that occurs in the early embryo, and that phosphorylation on this serine is required for the increased Dorsal nuclear import seen in ventral cells. This serine is absolutely conserved in all Rel proteins, and thus we propose that it serves a similar function in the other Rel family members.

\section{Results}

Dorsal is subject to signal-dependent phosphorylation while associated with Cactus in the cytoplasm

To determine in which cellular compartment the signaldependent Dorsal phosphorylation occurs, we constructed a constitutively cytoplasmic Dorsal protein by deleting its nuclear localization sequence ( $\Delta$ NLS). We measured the effects of this mutation on Dorsal function, using a dorsal minigene that results in highly reproducible expression of dorsal transgenes at levels indistinguishable from the endogenous locus (Table 1; Materials and Methods). The $\Delta N L S$-dorsal minigene was crossed into a dorsal-null background, and thus encodes the only source of Dorsal protein for the developing embryos. We monitored the phenotype, subcellular localization, and phosphoform distribution of this mutant protein in embryos from such transgenic females. The $\triangle N L S$-dorsal transgene produces embryos that show a severely dorsalized phenotype, and the protein is found exclusively in the cytoplasm at the blastoderm stage (Govind et al. 1996). Wild-type Dorsal forms a ventralto-dorsal nuclear gradient at this stage [see Fig. 6 below, wild type (WT), for an example]. Analysis by Western blot reveals that the $\Delta$ NLS-Dorsal protein from 0-3 hr embryos shows the same phosphoform distribution as wild-type Dorsal (Fig. 1A). 
Drier et al.

Table 1. Rescue analysis of putative Dorsal phosphorylation mutants

\begin{tabular}{|c|c|c|c|c|c|}
\hline Construct $^{\mathrm{a}}$ & $\begin{array}{c}\text { Copy } \\
\text { no. }\end{array}$ & $\begin{array}{l}\text { Hatch rate } \\
(\%)\left(25^{\circ} \mathrm{C}\right)^{\mathrm{b}}\end{array}$ & $\begin{array}{c}\text { Total } \\
\text { no. }\end{array}$ & $\begin{array}{c}\text { Cuticle } \\
\text { phenotype }^{\mathrm{c}}\end{array}$ & Protein distribution \\
\hline None & & 0 & $>300$ & D0 & no protein \\
\hline Endogenous dorsal allele & 1 & 47 & 303 & WT & WT gradient \\
\hline \multirow[t]{2}{*}{ Wild-type cDNA } & 1 & 53 & 423 & WT & WT gradient \\
\hline & 2 & 94 & 407 & WT & N.D. \\
\hline \multirow[t]{2}{*}{ 6XS-A } & 1 & 0 & $>300$ & D0 & cytoplasmic \\
\hline & 2 & 0 & $>300$ & D0 & cytoplasmic \\
\hline \multirow[t]{2}{*}{ S70A } & 1 & 0 & 401 & D3 & WT gradient \\
\hline & 2 & 14 & 265 & WT & N.D. \\
\hline \multirow[t]{2}{*}{ S79A } & 1 & 73 & 371 & WT & WT gradient \\
\hline & 2 & 90 & 261 & WT & N.D. \\
\hline \multirow[t]{2}{*}{ S103A } & 1 & 0 & 421 & D3 & WT gradient \\
\hline & 2 & 6 & 186 & WT & N.D. \\
\hline \multirow[t]{2}{*}{ S213A } & 1 & 0 & 340 & D3 & WT gradient \\
\hline & 2 & 9 & 343 & WT & N.D. \\
\hline \multirow[t]{3}{*}{ S312A } & 1 & 0 & 316 & D3 & WT gradient \\
\hline & & $3 \%\left(18^{\circ} \mathrm{C}\right)$ & 277 & D3 & \\
\hline & 2 & 12 & 329 & WT & N.D. \\
\hline \multirow[t]{2}{*}{ S317A } & 1 & 0 & $>300$ & D1 & decreased nuclear import \\
\hline & 2 & 0 & $>300$ & D2-D3 & decreased nuclear import \\
\hline T85A & 1 & 60 & 278 & WT & WT gradient \\
\hline $\mathrm{T} 290 \mathrm{~V}$ & 1 & 83 & 341 & WT & WT gradient \\
\hline Y324F & 1 & 90 & 320 & WT & WT gradient \\
\hline
\end{tabular}

We expressed the respective transgenes under the regulation of $\sim 4.5 \mathrm{~kb}$ of genomic DNA from the dorsal locus, which was immediately $5^{\prime}$ to the transcription unit (see Materials and Methods). We established three to four independent lines for each mutant and measured their ability to fully complement and phenotypically rescue the dorsal-null background. These results are taken from a single representative line from each construct, but transgenes under this regulation exhibit line-independent levels of expression; both phenotypes and hatch rates from individual lines of the same construct were indistinguishable (data not shown). The only variation we observed was an occasional line that showed no expression, presumably because of incorporation into heterochromatin. (WT) Wild type; (N.D.) not determined.

aThe endogenous dorsal allele is the wild-type allele on the $C y O b$ balancer chromosome of the $b \mathrm{dl}^{8} \mathrm{pr} \mathrm{cn} w \mathrm{w} b \mathrm{w} / \mathrm{CyO} b$ stock $(\mathrm{see}$ Drosophila stocks, in Materials and Methods).

${ }^{\mathrm{b}}$ All mutant transgenes were tested for temperature sensitivity, but only S312A was affected (see text).

cClassification of cuticle phenotypes is after Roth et al. (1991). (See Figs. 4 and 6 for photographs of representative cuticle phenotypes and Dorsal distribution.)

To determine whether Dorsal is phosphorylated before or after dissociating from Cactus, we used a cactus allele $\left(\right.$ cactus $\left.^{B Q}\right)$ encoding a protein that is recalcitrant to the signal. This mutant Cactus protein lacks the phosphorylation sites that target it for signal-dependent degradation and results in completely dorsalized embryos in which Dorsal is found exclusively in the cytoplasm, even in the presence of constitutive signaling (Roth et al. 1991; Bergmann et al. 1996). In this mutant background, we find that the hyperphosphorylated form of Dorsal is also clearly present (Fig. 1B). Taken together, these results show that a signal-dependent phosphorylation of Dorsal occurs in the cytoplasm while it is associated with Cactus. However, these results do not rule out the absence of additional phosphorylation events beyond the resolution of this technique (see Discussion).

\section{In vivo phospholabeling of Dorsal is restricted} to serine residues

Using phosphoamino acid analysis, we investigated whether the phosphorylation of Dorsal occurs on serine, threonine, or tyrosine residues. We labeled dissected ovaries by incubating them for $3 \mathrm{hr}$ in a phosphate-free insect cell culture medium to which $\left[{ }^{32} \mathrm{P}\right]$ orthophosphate was added. Embryonic labeling was done by direct injection of cleavage stage embryos with $\left[{ }^{32} \mathrm{P}\right]$ orthophosphate followed by the extraction of the embryonic contents after $\sim 2 \mathrm{hr}$ (at the cellular blastoderm stage, see Materials and Methods). Labeled Dorsal was isolated by immunoprecipitation with antibodies directed against Dorsal. The immunoprecipitates from wild-type extracts contain two prominent labeled bands that run at molecular weights that agree with Dorsal and Cactus (Fig. 2A). These bands are missing from parallel labelings of ovaries and embryos from females bearing protein-null mutations of dorsal (Fig. 2A), although these controls contained a similar level of ${ }^{32} \mathrm{P}$ incorporation into total protein (data not shown).

Phosphoamino acid analysis of a total acid-hydrolysate of Dorsal from both ovaries and embryos shows that labeling is restricted to serine residues, with no detectable incorporation into either tyrosine or threonine (Fig. 2B). The phosphoamino acid analysis of the labeled Cac- 


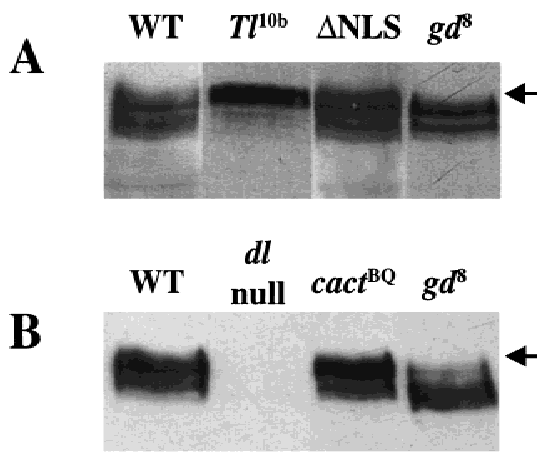

$\alpha$-Dorsal Western Blots

Figure 1. Dorsal is subject to signal-dependent phosphorylation while associated with Cactus in the cytoplasm. Extracts from 20 embryos were loaded in each lane. The genotype of the mothers is indicated. (Arrow) Location of the hyperphosphorylated form of Dorsal in $A$ and $B$. (A) Constitutively cytoplasmic $\triangle$ NLS-Dorsal is phosphorylated normally. (WT) Mothers are homozygous wild-type for dorsal and the dorsal-ventral signal transduction pathway. $\left(T 1^{10}\right)$ Mothers bear a dominant allele of the gene encoding the transmembrane receptor Toll, which results in constitutive signaling and causes Dorsal to translocate to all nuclei along the dorsal-ventral axis at a high, uniform level (Roth et al. 1989; Steward 1989). (DNLS) Mothers bear one copy of the $\triangle N L S$-dorsal mutant minigene in an otherwise dorsal protein-null mutant background. $\left(g d^{8}\right)$ Mothers are homozygous for $g d^{8}$ that completely disrupts signaling and causes Dorsal to remain cytoplasmic along the entire dorsal-ventral axis (Roth et al. 1989; Steward 1989). (B) Dorsal is phosphorylated when associated with Cactus. (WT) The same as in $A$. (dl-null) Mothers bear two different protein-null alleles of dorsal. $\left(\right.$ cact $\left.^{B Q}\right)$ The mothers genotype is cact $^{B Q} /$ cact $^{E 10 R N 1}$. cact $^{B Q}$ is an allele of cactus that is completely recalcitrant to the ventral signal and thus constitutively maintains Dorsal in the cytoplasm (Bergmann et al. 1996). cact $^{E 1 O R N 1}$ is a null allele of cactus (Roth et al. 1991). $\left(g d^{8}\right)$ The same as in $A$.

tus shows that it too is labeled exclusively on serine (data not shown). Serine phosphorylation has been shown to be important for dissociation of the DorsalCactus complex (Reach et al. 1996); however, it is not clear what role is played by the phosphorylation of Cactus in the complex.

\section{A $6 X$ serine-to-alanine mutation abolishes Dorsal activity}

A Dorsal RHR-LacZ fusion protein produces a gradient in early embryos, demonstrating that the RHR contains all the regions necessary for nuclear targeting. This observation along with other work shows that the RHR contains sequences essential for dimerization, DNA binding; Cactus, Tube, and Pelle interaction; and signaldependent nuclear translocation (Ip et al. 1991; Govind et al. 1996; Edwards et al. 1997; Yang and Steward 1997). As the labeling of Dorsal is limited to serine, we focused on six serines within the RHR that show strong conservation within the Rel family (Fig. 3; Materials and Methods). To eliminate phosphorylation of these serines, a mutant changing all six serines to alanine was made by site-directed mutagenesis (6XS-A-Dorsal) and transgenic lines expressing this mutant protein were established.

We crossed the $6 X S$ - $A$-dorsal minigene into the dorsal-null background. The 6XS-A mutant fails to rescue (Table 1; Fig. 4C), and the embryonic phenotype is indistinguishable from the dorsal-null controls (Table 1; Fig. 4B), whereas one copy of the wild-type dorsal minigene functions in the dorsal-null background to the same degree as an endogenous wild-type allele (Table 1; Fig. 4A). This result demonstrates that the 6XS-A mutation abolishes Dorsal function.

\section{XS-A-Dorsal is constitutively cytoplasmic}

The complete loss of function of the 6XS-A-Dorsal could either be due to a defect in nuclear targeting, or to the elimination of Dorsal's transcriptional activity. To distinguish between these two possibilities, we stained early embryos using anti-Dorsal antibodies. Figure 4D shows that 6XS-A-Dorsal protein is clearly present but

\section{A}

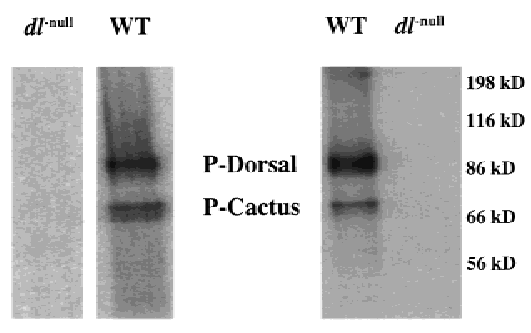

\section{B}
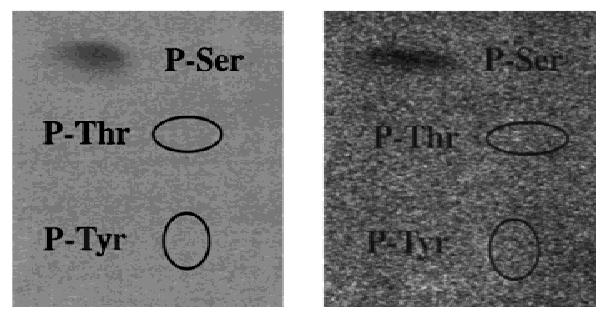

Figure 2. In vivo phospholabeling of Dorsal is limited to serine residues. (A) SDS-PAGE of labeled Dorsal from both ovaries (left) and early embryos (right). Extracts were made from labeled ovaries and embryos (see Materials and Methods). Dorsal was immunoprecipitated with pooled anti-Dorsal monoclonal antibodies that were conjugated to protein A-Sepharose beads. Parallel labelings and immunoprecipitations were done on ovaries and embryos from mothers bearing protein-null mutations in dorsal. These dorsal-null labelings had a similar level of total protein phosphorylation (data not shown). (B) Phosphoamino acid analysis of ovarian (left) and embryonic (right) Dorsal. The labeled Dorsal from $A$ was transferred to Immobilon-P membrane and subjected to total hydrolysis in $5.7 \mathrm{~N} \mathrm{HCl}$ (see Materials and Methods). The hydrolysates were separated by TLC. The ellipses indicate the locations of the ninhydrin-stained PThr and P-Tyr. 


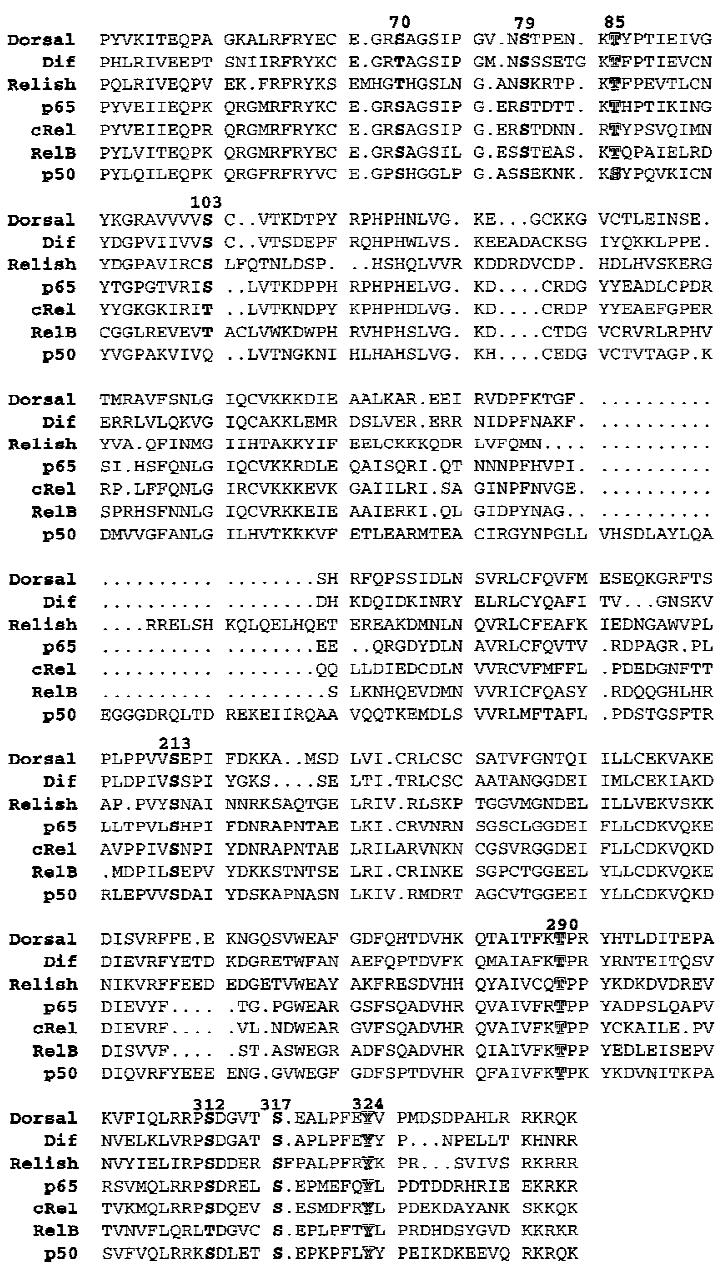

Figure 3. Conserved serines in the Rel homology domain of Dorsal. The Rel homology domain of Dorsal is compared with the other Drosophila Rel family members, Dif and Relish, as well as vertebrate forms, p65, c-Rel, RelB, and p50. The conserved serines are highlighted in bold. The conserved threonine and tyrosine residues we altered are indicated by shadowed characters. The bold numbers above the Dorsal sequence refer to the location of the residue within Dorsal.

remains cytoplasmic, indicating that it fails to respond to signals regulating Dorsal nuclear translocation. In the negative control, no Dorsal is detected in the dorsalmutant background without a dorsal transgene (see Fig. 6 below, dl-null), whereas a wild-type dorsal minigene produces a normal nuclear gradient (see Fig. 6 below, WT).

To test whether the 6XS-A mutant fails to dissociate from Cactus, or when free, remains unable to undergo nuclear translocation, we crossed the mutant transgene into a cactus $^{A 2}$, dorsal-null background. Embryos from these mutant mothers are completely dorsalized (identical to Fig. 4C; data not shown), and 6XS-A-Dorsal remains cytoplasmic (identical to Fig. $4 \mathrm{D}$; data not shown). We further tested whether the 6XS-A protein responds to enhanced signaling as it occurs in a constitutively active allele of Toll, that causes wild-type Dorsal to translocate to nuclei at ventral levels along the entire dorsal-ventral axis. Embryos from $\mathrm{Toll}^{10 b}$ females relying exclusively on the 6XS-A protein for Dorsal activity are also completely dorsalized and the protein remains cytoplasmic (as in Figs. 4C,D; data not shown).

\section{The 6XS-A protein eliminates Dorsal phosphorylation}

The distribution of Dorsal phosphoforms can be monitored by Western blots of low-bis acrylamide gels (see Materials and Methods; Whalen et al. 1993; Gillespie et al. 1994). A low-bis Western blot shows that although the levels of the 6XS-A protein are comparable with wild type, the phosphoform distribution is clearly altered (Fig. 5A). The 6XS-A protein always migrates as a single band, whereas the wild-type form of Dorsal typically migrates as three to five distinct isoforms on these gels. The 6XS-A protein comigrates with bacterially expressed Dorsal and the most rapidly migrating of the wild-type Dorsal isoform. The 6XS-A protein band also coincides with the embryonic Dorsal protein subjected to dephosphorylation (WT de-P). The 6XS-A protein from ovaries
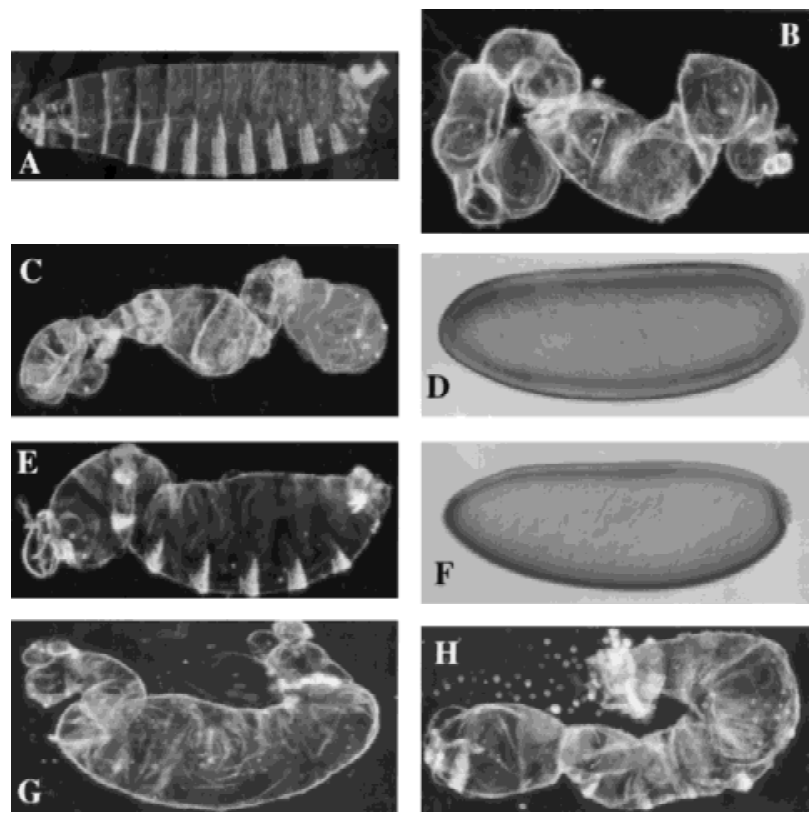

Figure 4. Molecular and phenotypic analyses of S-to-A Dorsal mutants. Cuticle preparations of fully differentiated embryos and anti-Dorsal staining of blastoderm stage embryos. (A) An embryo from a mother bearing two copies of a wild-type dorsal cDNA. (B) An embryo from a dorsal-null mother without a dorsal minigene. (The dorsal-null background used throughout this work; see Materials and Methods). (C) An embryo from a mother bearing two copies of $6 X S$-A-dorsal in the dorsal-null background. $(D)$ Anti-Dorsal staining of a blastoderm embryo as in C. (E) An embryo from a mother bearing one copy of S312Adorsal in the dorsal-null background. $(F)$ Anti-Dorsal staining of a blastoderm embryo as in $E$. $(G, H)$ Embryos from mothers bearing one or two copies of $S 317 A$-dorsal in the dorsal-null background, respectively. See Fig. 6 for anti-Dorsal staining of wildtype Dorsal, dorsal-null, and S317A-Dorsal. 
A

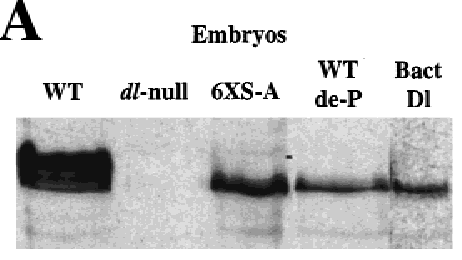

$\alpha$-Dorsal Western
Ovaries
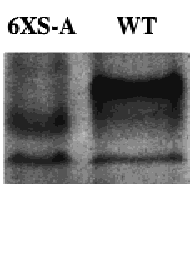

Embryos

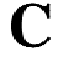

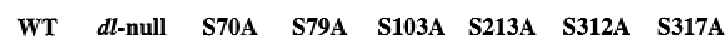

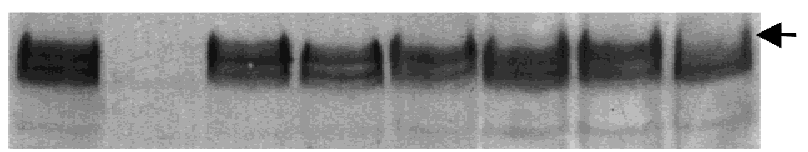

$\alpha$-Dorsal Western

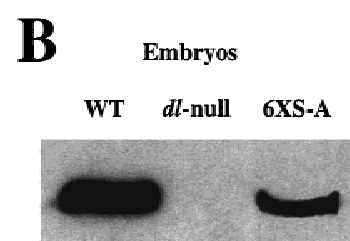

$\alpha$-Cactus Western

Figure 5. Western analyses of Dorsal serine-toalanine mutations. $(A)$ The 6XS-A mutant eliminates Dorsal phosphoforms. A low-bis Western blot probed with anti-Dorsal antibody. (Left) Embryos. Approximately thirty, 0 to $3 \mathrm{hr}$ embryos were used for each lane. The lane markings indicate the origin of the Dorsal protein in each case. The 6XS-A comes from dorsal-null mothers bearing two copies of the $6 X S$-A-dorsal minigene. (WT de-P) Dephosphorylated wildtype Dorsal (see Materials and Methods). (BactDl) Bacterially expressed Dorsal. (Right) Ovaries. One pair of ovaries was used for each lane. $(B)$ The 6XS-A-Dorsal mutant protein interacts with Cactus. A Western blot probed with antiCactus antibody. The 6XS-A females carried only one copy of the 6XS-A-dorsal minigene, whereas the wild-type (WT) lane carries two endogenous dorsal copies. (C) The phosphoform distribution of single serine-to-alanine mutations. A representative low-bis Western blot probed with anti-Dorsal antibody. In each case, the embryos were from mothers bearing two copies of the respective transgenes. The subtle changes in phosphoform distribution between each serine-to-alanine mutant are somewhat variable. The S317A mutant differs consistently from both wild type and the other mutants.

has the same mobility as the embryonic (Fig. 5A, ovaries). These results indicate that the $6 \mathrm{XS}-\mathrm{A}$ mutation eliminates Dorsal phosphorylation during oogenesis as well as embryogenesis.

To assess the structural integrity of the 6XS-A protein, we tested its ability to interact with Cactus. We took advantage of the fact that Dorsal is required to stabilize Cactus in the early embryo (Whalen et al. 1993; Belvin et al. 1995), suggested by the observation that in a dorsalnull background, Cactus cannot be detected by Western analysis (Fig. 5B, dl-null). However, Cactus is present at levels comparable with the wild-type control in embryos containing the 6XS-A mutant as the only Dorsal protein available (Fig. 5B, 6XS-A). This observation demonstrates that, like wild-type Dorsal, the 6XS-A protein is able to interact with Cactus, indicating that these mutations do not cause severe disruptions of Dorsal's structure.

\section{Analysis of single serine-to-alanine mutations in a dorsal-null background}

To further characterize the importance of Dorsal phosphorylation, we mutated each of the six serines to alanine individually and examined their activity in the dorsal-null background. The $S 79 A$-dorsal minigene functions indistinguishably from wild-type dorsal in all our measurements (Table 1). Four single serine-to-alanine mutants (S70A, S103A, S213A, and S312A) have indistinguishable effects in the dorsal-null background. Table 1 shows that each minigene fails to rescue the dorsalnull background to hatching when present in a single copy but will support a low level of hatching when present in two copies. Embryos from a mother carrying one copy of each minigene in a dorsal null background are weakly dorsalized (S312A-dorsal is shown, Fig. 4E) and the proteins form qualitatively normal nuclear gradients (S312A-Dorsal is shown, Fig. 4F; see Fig. 6, WT, for the wild-type Dorsal gradient), as expected from the hatchrate analysis.

The mutation of serine 317 to alanine (S317A-Dorsal) severely reduced the function of the Dorsal protein. Embryos from females carrying one copy of the S317A-dorsal in a dorsal-null background display a strongly dorsalized phenotype (Table 1; Fig. 4G), whereas females that carry two copies produce more weakly dorsalized embryos (Table 1; Fig. 4H). Even in two copies, the phenotype of $S 317 A$-dorsal is stronger than that of the other mutants in one copy, and they do not hatch.

We determined whether any single serine-to-alanine mutation produced detectable effects on Dorsal phosphoform distribution by low-bis Western blot. Figure 5C shows a representative Western of these mutants. Although all of the mutants produce similar levels of protein, only S317A-Dorsal shows a reproducible change in phosphoform distribution. This mutant completely lacks the hyperphosphorylated form. Interestingly, the phosphoform distribution of S317A-Dorsal looks similar to that of wild-type-Dorsal in a gastrulation defective $(g d)$ mutant background, in which the signal is disrupted (see Fig. 1, $\left.g d^{8}\right)$. Although we cannot resolve any phosphoform changes in the remaining single phosphorylation-site mutations, the fact that the 6XS-A-Dorsal is not phosphorylated indicates that at least some subset of these other sites represent in vivo targets of phosphorylation that cannot be detected by Western blot.

\section{S312 phosphorylation is required to stabilize Dorsal} in the absence of Cactus

In an attempt to determine whether the loss-of-function phenotypes observed in the S-A mutations is due to their failure to target normally to the nucleus, or to the lossof-function as a transcription factor, we crossed each S-A mutation into the cactus $^{A 2}$, dorsal-null double homozy- 

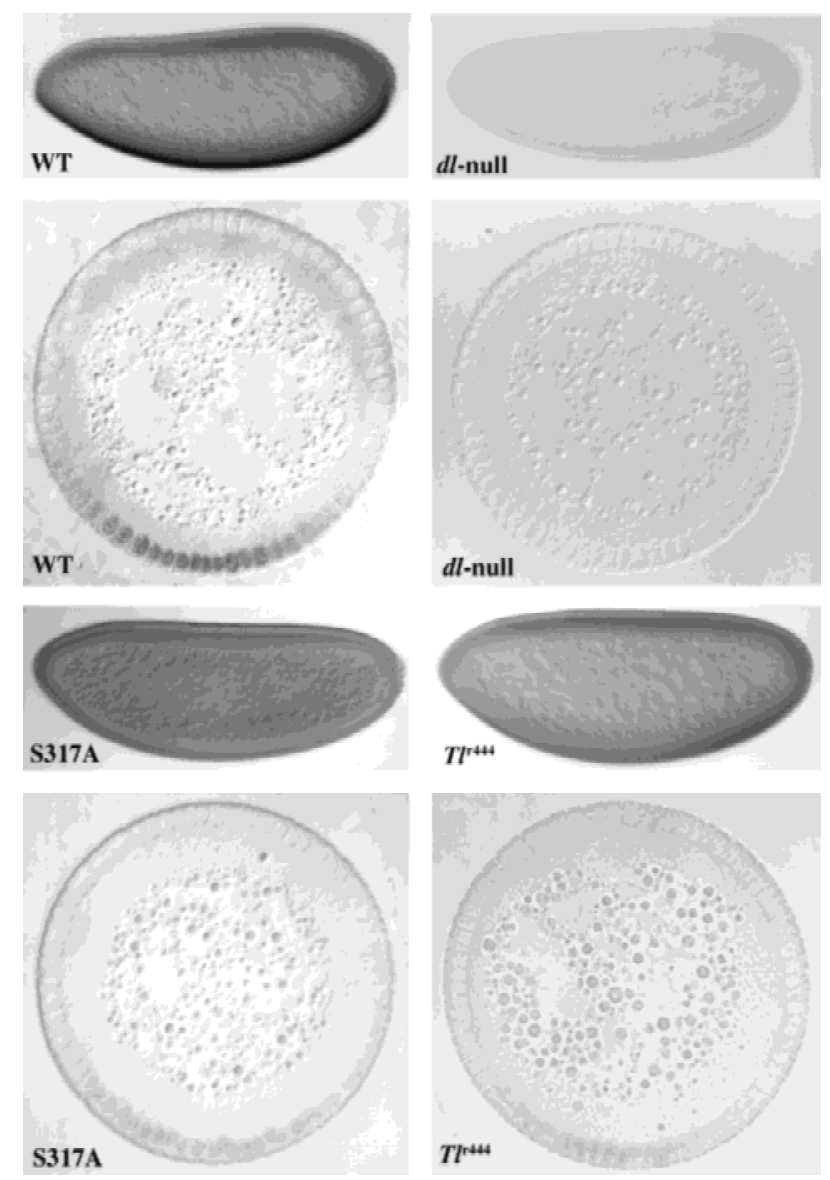

Figure 6. Anti-Dorsal stainings of embryos show that S317ADorsal is defective for normal nuclear import. Whole mounts and cross sections of blastoderm stage embryos stained with pooled anti-Dorsal monoclonals (see Materials and Methods). In the whole mounts, anterior is to the left and dorsal is up; in the cross sections, dorsal is up. The cross sections were taken at roughly the anterior-posterior midpoint of the embryo. (WT) Embryos from mother bearing one copy of the wild-type dorsal minigene in the dorsal null background. (dl-null) Embryos from mothers bearing protein-null mutations in dorsal (see Materials and Methods) without any dorsal minigene. (S317A) Embryos from mothers bearing one copy of the $S 317 A$-dorsal mutant minigene instead of the wild-type minigene. $\left(T 7^{r 444}\right)$ Embryos from a Toll ${ }^{444}$ mother [genotype: $T 1^{r 444} / D f(T 1)$; see Materials and Methods] raised at the restrictive temperature $\left(29^{\circ} \mathrm{C}\right)$.

gous background. In this background, mothers bearing the wild-type dorsal minigene produce strongly ventralized embryos that do not hatch, and Dorsal is distributed in a dorsally extended gradient (Fig. 7A,C,E) (Roth et al. 1989; Steward 1989). As expected, S79A-dorsal retains full function and behaves indistinguishably from wildtype dorsal in this background (data not shown). Embryos from S70A-dorsal, S103A-dorsal, and S213A-dorsal mothers also show indistinguishable ventralized phenotypes in this background, similar to that produced by the wild-type dorsal minigene (S70A-dorsal represents this class, Fig. 7G). These mutant proteins are distributed in a dorsally extended nuclear gradient in blasto- derm stage embryos, qualitatively indistinguishable to that observed in the wild-type control (Fig. 7; cf. C with I).
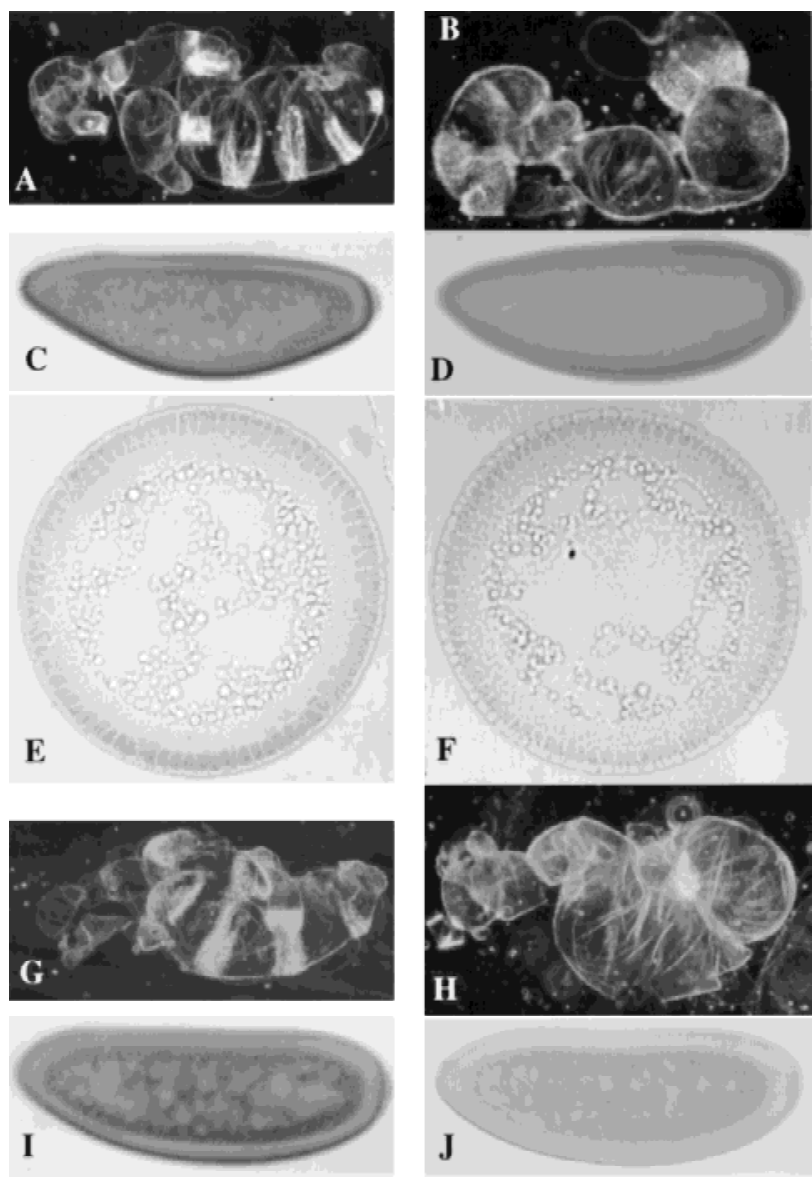

Figure 7. S317A-Dorsal is uniformly distributed and S312ADorsal in unstable in a cactus $^{A 2}$, dorsal-null mutant background. Cuticle preparations of fully differentiated embryos and blastoderm stage embryos stained with pooled anti-Dorsal monoclonals (see Materials and Methods). (A) A cuticle preparation of an embryo from a mother bearing one copy of the wild-type dorsal minigene in a cact $^{A 2}$, dorsal-null background. (B) The same as in $A$, except the mother carries one copy of the S317A-dorsal minigene instead of the wild-type minigene. $(C)$ A whole mount antibody staining of an embryo from a mother bearing one copy of the wild-type dorsal minigene in a $\mathrm{cact}^{A 2}$, dorsal-null background. $(D)$ the same as in $C$, except the mother carries one copy of the S317A-dorsal minigene instead of the wild-type minigene. The nuclei are difficult to visualize in $D$ because S317A-Dorsal does not make a nuclear gradient as seen in $C$, and stains the nuclei and cytoplasm at roughly equal levels. (E) A cross section of an embryo as in C. (F) A cross section of an embryo as in $D$, which more readily shows the uniform low level of S317A-Dorsal entering the nuclei. In this cross section, we cannot state that dorsal is up because the S317ADorsal shows no gradient to indicate dorsal-ventral polarity. $(G, H)$ Cuticle preparations of embryos from a mother bearing one copy of S70A-dorsal. (G) One copy of S312A-dorsal in the cactus $^{A 2}$, dorsal-null background. $(I, J)$ Whole mount anti-Dorsal stainings of blastoderm stage embryos as in $G$ and $H$, respectively. 
In contrast, mothers bearing the $S 312 A$-dorsal minigene give rise to severely dorsalized embryos (D1, Fig. $7 \mathrm{H})$. This surprising result is explained by the S312ADorsal staining in blastoderm stage embryos. We find that this protein is unstable in the cactus $^{A 2}$, dorsal-null background; virtually no protein is detected (Fig. 7, cf. J: S312A-Dorsal with C: wild-type Dorsal and I: S70ADorsal).

\section{Signal-dependent phosphorylation of S317 regulates Dorsal nuclear import}

The Western analysis combined with the phenotypic consequences of the S317A mutation show that S317 is phosphorylated in the embryo and that this phosphorylation is critical for Dorsal function. Therefore, we did a detailed analysis of the distribution of this mutant protein. Mutant blastoderm stage embryos stained with anti-Dorsal antibody show a clear and reproducable reduction of the level of nuclear protein (Fig. 6). This observation is supported by both whole mounts and cross sections of stained blastoderm stage embryos, in which the amount of nuclear S317A-Dorsal is significantly lower than that observed in wild-type embryos (Fig. 6, cf. WT and S317A, whole mount and cross section).

The strongly dorsalized phenotype observed in S317A embryos is also seen in embryos from females with lossof-function mutations in genes that are part of the dorsal-ventral signaling pathway. Toll encodes the transmembrane receptor of the pathway and embryos from mothers bearing a hypomorphic allele of Toll (Toll ${ }^{\text {r444}}$ ) over a deletion show, at restrictive temperature, a phenotype indistinguishable from that of one copy of the S317A-dorsal (Schneider et al. 1991). The reduction of Toll activity in Toll ${ }^{r 44}$ results in a low level of nuclear import of Dorsal and the embryos show only a restricted Dorsal nuclear gradient in blastoderm stage embryos, similar to that observed in embryos from S317A-dorsal females (Fig. 6, cf. S317A and $T^{r 444}$ to each other and WT, whole mount and cross section). These results indicate that S317A-Dorsal is unable to respond properly to the ventral signal.

For Dorsal to target to the nucleus, the protein must first be freed from its interaction with Cactus. To determine whether the nuclear localization defect of S317ADorsal is due to a failure to dissociate from Cactus, we compared its function and distribution with wild-type Dorsal in a cactus mutant background. In distinct contrast to the other single serine-to-alanine mutants and the wild-type dorsal minigene, cactus $^{A 2}$, dorsal-null females bearing the $S 317 A$-dorsal mutant transgene give rise to lateralized, rather than ventralized, embryos (Fig. 7, cf. B with A and G). Analysis of Dorsal protein distribution in these embryos is in complete agreement with these cuticle phenotypes. S317A-Dorsal targets to the nuclei along the entire dorsal-ventral axis but does so at a uniform and low level, equivalent to that observed in lateral nuclei in a wild-type embryo (Fig. 7D and F, whole mount and cross section, respectively). In contrast, the wild-type Dorsal protein produces a gradient that is extended dorsally (Fig. 7C and E, whole mount and cross section, respectively) (Roth et al. 1989; Steward 1989). Because its resulting phenotype and protein distribution are clearly different in the cactus $^{A 2}$ background compared with a wild-type cactus background, the defect of S317A-Dorsal is not the result of a failure to dissociate normally from Cactus. Thus, S317A-Dorsal is unique among our putative phosphorylation mutants: Although it is able to target to nuclei at a basal, lateral level, it clearly does not translocate at levels normally seen in ventral nuclei. These results strongly suggest that it lacks the ability to appropriately respond to the ventral signal.

\section{Discussion}

Cytoplasmic Dorsal phosphorylation and the ventral signal

We have explored the relationship between Dorsal phosphorylation and its regulated nuclear import. Previous work has shown that Dorsal is multiply phosphorylated and that the hyperphosphorylated form correlates with its nuclear import (Whalen et al. 1993; Gillespie et al. 1994). However, it was not clear whether this phosphorylation was a cause, or effect, of Dorsal import.

We show that the cytoplasmically localized $\Delta$ NLSDorsal mutant is subject to signal-dependent phosphorylation. We further show that wild-type Dorsal, maintained in the cytoplasm by a mutant Cactus protein that is recalcitrant to the signal (Cactus ${ }^{\mathrm{BQ}}$ ), is also subject to this same phosphorylation. These results show that a signal-dependent Dorsal phosphorylation occurs in the cytoplasm, whereas Dorsal is bound to Cactus.

Gillespie and Wasserman (1994) monitored Dorsal phosphorylation in a double-mutant background, which disrupts both the signal and Cactus (signal-null, cactus $\left.^{A 2}\right)$. In this background, Dorsal translocates to nuclei at a uniform, lateral level along the entire dorsal-ventral axis. The resulting embryos are lateralized rather than ventralized, further indicating a direct effect of the ventral signal on Dorsal nuclear import (Roth et al. 1991). Interestingly, they found that the hyperphosphorylated form of Dorsal is present in this background, but it is less abundant than in the presence of the signal (Gillespie et al. 1994). This hyperphosphorylated form is missing in a signal-null, cactus wild-type background. The presence of this phosphoform in the absence of the signal is only consistent with a model in which a constitutively active kinase modifies Dorsal but only when it is dissociated from Cactus.

When combined with our current results, it is likely that the slowest migrating Dorsal band actually contains more than one Dorsal phosphoform. One form represents the signal-dependent phosphorylation of Dorsal in the cytoplasm, while bound to Cactus. The other form represents the activity of a constitutive kinase that targets free Dorsal. This constitutive kinase could function in the cytoplasm or in the nucleus. Both the $\Delta$ NLS-Dorsal and the Dorsal bound to Cactus ${ }^{\mathrm{BQ}}$ apparently contain 
all phosphoforms, but the absence of a band may be masked. Our results show that Dorsal is subject to at least one signal-dependent phosphorylation while bound to Cactus in the cytoplasm, and thus this event is a potential regulator of Dorsal nuclear import.

\section{Phosphorylation of S317 controls Dorsal nuclear import}

Our in vivo labeling shows that detectable phosphorylation of Dorsal in both ovaries and embryos is limited to serine residues. The $6 \mathrm{XS}-\mathrm{A}-\mathrm{Dorsal}$ protein migrates as a single band on Western blots, which comigrates with dephosphorylated Dorsal. The lack of phosphorylation of this mutant is unlikely the result of gross structural defects, because this protein retains its Cactus-binding activity. Thus, we infer that 6XS-A-Dorsal is mutant at in vivo phosphorylation sites, and that this mutant is defective in most, if not all, Dorsal phosphorylation. Although all but one (S79A) of the single serine-to-alanine mutants have measurable effects on Dorsal activity, only S317A-Dorsal produces a measurable effect on Dorsal phosphorylation (see Fig. 5).

We have shown that S317A-Dorsal is unique among our single serine-to-alanine mutations in three respects: First, it clearly alters the phosphoform distribution of Dorsal, and is notably missing the hyperphosphorylated form; second, it significantly reduces the nuclear targeting of Dorsal in a dorsal-null background; and finally, in a cactus $^{A 2}$, dorsal-null background, it gives rise to lateralized rather than ventralized embryos, and undergoes nuclear import at uniform low levels rather than forming a dorsally extended nuclear gradient. We emphasize that these conclusions are based on Dorsal phosphoform distributions on Western blots, as well as embryonic phenotypes and protein localization in antibody stained embryos. In all cases, the embryonic cuticular phenotype is in complete agreement with the results obtained from embryo stainings.

The S317A mutation does not eliminate Dorsal nuclear import, as is observed in a signal-null background. There is a fundamental difference in these two situations: In a signal-null background, neither Cactus nor Dorsal are modified, whereas in the S317A mutant, Cactus modification and degradation is likely to proceed normally, but Dorsal does not respond to the signal. The phenotype and Dorsal protein distribution observed from the S317A mutant in the cactus $^{A 2}$, dorsal-null background are similar to those observed for wild-type Dorsal in a mutant background, in which both cactus and signaling are disrupted (Roth et al. 1991). In that case, Dorsal does not achieve ventral levels of nuclear import because there is no signal. The S317A mutant results in the same phenotype and protein distibution because it lacks the Dorsal-intrinsic target of the signaling system. Thus, we propose that the hyperphosphorylated form of Dorsal is due to the signal-dependent phosphorylation of S317, and that this modification controls the high level nuclear targeting of Dorsal in ventral cells.

Our results further indicate that the basal level of nuclear import seen in the S317A mutant also requires Dorsal phosphorylation. The 6XS-A completely fails to translocate in response to wild-type or constitutive signaling $\left(T_{0} 1 l^{10 b}\right)$, as well as in backgrounds that substantially reduce Cactus activity (cactus ${ }^{A 2}$ ). The 6XS-A mutant appears to eliminate all Dorsal phosphorylation; but it remains able to interact with Cactus and thus is unlikely to be severely misfolded. In contrast, S317A-Dorsal, which translocates to the nuclei at basal levels, remains subject to the signal-independent phosphorylation, because it still produces several slower running phosphoforms as observed with wild-type Dorsal in the absence of signal. We conclude that signal-independent phosphorylation of Dorsal is essential for the basal level of nuclear import, and that in response to the ventral signal, phosphorylation on S317 results in a normal nuclear gradient.

\section{S312 phosphorylation and Dorsal stability}

S312 is the putative phosphoacceptor of a PKA site that is highly conserved within the Rel family. Previous work has implicated S312 phosphorylation in the control of both nuclear localization and transcriptional activity in tissue culture. In transient transfections of Drosophila tissue culture cells, Norris and Manley (1992) showed that Dorsal nuclear transport and transcriptional activity were augmented by cotransfected Toll receptor and/or cotransfected PKA. Briggs et al. (1998) corroborated these results in rat hepatoma cells, and used an in vitro direct binding assay to show that the importin 58/97 complex recognition of the Dorsal NLS was influenced by the integrity of the PKA site. Neither of these studies showed that S312 was actually phosphorylated, nor do we have direct evidence that S312 is phosphorylated in vivo.

All of our work was done with a dorsal minigene that behaves, in all measurements, as a single copy of an endogenous dorsal allele. We find that S312A-Dorsal retains most of its function, and that its distribution appears normal in a dorsal-null background. Notably, Briggs et al. (1998) also found that a S312A-dorsal transgene expressed under the control of the bicoid promoter forms a qualitatively normal nuclear gradient, and supports substantial embryonic dorsal-ventral patterning, approaching the phenotype we observe with the S312Adorsal minigene. Thus, when measured in vivo, S312 plays a subtle, or perhaps redundant role in regulating Dorsal nuclear import. However, we find that S312 is clearly involved in controlling Dorsal stability. This function is best revealed in the cactus ${ }^{A 2}$, dorsal-null background, in which the S312A-Dorsal results in completely dorsalized embryos and almost no protein is detected (Fig. 7J). Interestingly, an EMS allele, dorsal ${ }^{P Z}$, maps to the PKA and shows a similar dorsal loss-offunction phenotype in a cactus ${ }^{A 2}$ background (Isoda et al. 1992). In dorsal $P^{P Z}$, the serine remains intact, and an adjacent arginine is changed to a histidine (R310H). This mutant protein can be detected on a Western blot of extracts from embryos raised at $18^{\circ} \mathrm{C}$, but the protein is 
destabilized in embryos raised at $25^{\circ} \mathrm{C}$. We tested each of our serine-to-alanine mutants for temperature sensitivity and only S312A-Dorsal improves at $18^{\circ} \mathrm{C}$ (Table 1). Hence, the weak loss-of-function phenotype of S312ADorsal observed in the dorsal-null background may also be due to a reduction in the stability of the protein.

Phosphorylation of the corresponding serine in NF- $\mathrm{B}$ p65 was shown to be involved in stimulating transcription and regulating the association of NF- $\mathrm{kB}$ p 65 with a coactivator CBP/p300 (Zhong et al. 1998). Our results are not inconsistent with these findings; they suggest that phosphorylation of the PKA site does not control specific functions of the Rel protein, rather that the PKA site is essential for the stability of the proteins. Although our in vivo assay is highly sensitive to levels of active Dorsal protein, the results in tissue culture were obtained in transient assays, in which instability of the protein would be more difficult to detect. It is also possible that the S312A mutation behaves differently in vivo than in tissue culture.

The fact that the stability of S312A-Dorsal is much more strongly affected when Cactus is missing suggests that on dissociation of Dorsal from Cactus, it is stabilized by the interaction with Tube or Pelle (Edwards et al. 1997; Yang et al. 1997), or by interaction with a protein that controls the nuclear import of Dorsal. Whereas S312A-Dorsal is unstable in the absence of Cactus, the nonphosphorylated 6XS-A protein is present in the same background. This apparent contradiction can only be explained by a requirement of phosphorylation on at least one of the remaining serines for the degradation of Dorsal.

\section{Dorsal phosphorylation and transcriptional activity}

Dorsal can both activate and repress transcription (Jiang et al. 1991, 1993; Kirov et al. 1993). We monitored the expression of snail, which is activated, and $d p p$, which is repressed by Dorsal, in wild-type and the serine-to-alanine mutant backgrounds to determine whether any mutant produced a specific effect on Dorsal's activation or repression functions. We found a change of expression of both genes commensurate with the loss-of-function phenotype of each serine-to-alanine mutation, and in no case did any mutant clearly show a preferential affect on either activation or repression of these target genes (results not shown).

Although the expression of twist and snail does not overlap with that of decapentaplegic $(d p p)$ along the dorsal-ventral axis in the main body of the embryo, all three genes are activated at the embryonic termini. The Dorsal nuclear gradient extends around each terminus and thus its repressor function must be countered to achieve the polar expression of $d p p$. This derepression of $d p p$ requires the activity of the terminal-group RTK signal transduction (Ray et al. 1991). Two explanations have been proposed for this observation: The terminal-group signaling system may antagonize the repressor function of Dorsal by direct modification of Dorsal, which specifically alters it's ability to interact with its neighboring corepressor(s); alternatively, this regulation could occur by the modification of the corepressor(s) (Rusch and Levine 1994). Our single serine-to-alanine mutants show normal expression of $d p p$ at the poles (data not shown), indicating that terminal-group-mediated derepression occurs through the modification of the corepressor(s). Groucho has been shown to be key component of Dorsal repression, and thus may prove to be the direct target of the terminal-group signaling system (Dubnicoff et al. 1997).

\section{Potential kinases involved in Dorsal regulation}

Two of the serines that we have changed to alanines are predicted to be phosphorylated by specific kinases. S79 is part of a predicted casein kinase II (CK II) site, and interestingly, this is the only mutant that retains wild-type function, suggesting that if the serine is phosphorylated, the modification is not essential. S312 is part of a PKA recognition site. This site may or may not be phosphorylated, but it is not the target of the signal-dependent phosphorylation that occurs on Dorsal. Rather, if S312 is phosphorylated, the modification may occur already in the ovary, and stabilizes the protein, as discussed above.

We find that a serine (S317) that is not part of any known kinase recognition site is the likely target of the signal-dependent phosphorylation. This raises the question as to what kinase is responsible for the modification. Tube and Pelle are components of the dorsal-ventral signal transduction pathway that function downstream from the Toll receptor. They interact directly with Dorsal, albeit in the amino-terminal domain 1 of the RHR. This interaction represents an essential step in the transduction of the ventral signal (Edwards et al. 1997; Yang et al. 1997). Pelle is a serine-threonine kinase and may be directly responsible for the phosphorylation of Dorsal. However, as S317 is in domain 2 of the RHR, it is possible that additional kinases, such as the IкB kinases, phosphorylate Dorsal and that Pelle controls their activity (DiDonato et al. 1997; Mercurio et al. 1997; Woronicz et al. 1997; Zandi et al. 1997).

\section{Dorsal phosphorylation is limited to conserved serine residues within the Rel homology domain}

The 6XS-A mutant protein was designed to disrupt phosphorylation events at highly conserved serine residues within the RHR. This protein appears to eliminate all Dorsal phosphorylation. This result suggests that, at least in Dorsal, the function of the carboxy-terminal nonconserved part of the protein is not regulated by phosphorylation. To further rule out phosphorylation on other amino acids in the RHR, we also made mutations in two highly conserved threonine (T85A and T290V), and in one tyrosine (Y324F, see Fig. 3 and Materials and Methods). These mutations produce no measurable effects on Dorsal activity, each behaving indistinguishably from a wild-type dorsal cDNA (see Table 1).

The structure of a p50 RHR homodimer bound to a $\kappa \mathrm{B}$ 
site has been solved by X-ray crystallography (Ghosh et al. 1995; Muller et al. 1995). Although the serines we have altered are spread throughout the primary structure of the Dorsal RHR, the corresponding residues are rather close in the tertiary structure of p50. Five of the six serines in the p50 structure are located on the surface of the molecule, accessible for kinase recognition, and reside within the cleft formed between domain 1 and domain 2. S103 is the least conserved of the serines (Q95 in p50) and is buried within domain 1 of the RHR. The position of S312 (S335 in p50) is in close proximity to other amino acids with which it could possibly form hydrogen bonds.

The conservation of the serine residues and their location within the p50 structure together imply that the effects of serine phosphorylation will not be limited to Dorsal. We propose that the existing model of the control of nuclear localization of Rel proteins requires amendment: Activation of the Rel pathway in Drosophila and vertebrates results in the phosphorylation of both the IкB and the Rel proteins, and that both modifications are essential for the proper nuclear targeting of the Rel protein.

\section{Materials and methods}

Drosophila stocks

al $d p b \operatorname{Df}(2 L) T W 119 \mathrm{cn} / \mathrm{CyO} b$ (this deficiency removes $d l$ ) and $b$ pr cn $d l^{8} w x^{w x t} b w / C y O b$ (this is a protein-null allele of $d l$ ) and $b$ pr cn sca $\operatorname{In}(2 L) d l^{H} / C y O b$ (this inversion breaks within d1) (Steward and Nüsslein-Volhard 1986) were used to create the dorsal-null background. $b$ cact $^{A 2} d 1^{1} / C y O b$ was used for the cactus-dorsal double-mutant background. $d l^{1}$ is a protein-null allele (Isoda et al. 1992) and cact $^{A 2}$ is the strongest viable allele of cactus. Homozygous cact ${ }^{A 2}$ was used as the cactus minus background (Roth et al. 1991). $\mathrm{Tl}^{10 b}$ is a dominant, constitutively active allele of Toll (Erdelyi and Szabad 1989) and $\mathrm{Tl}^{\mathrm{r} 444}$ is a temperature-sensitive, hypomorphic allele (Schneider et al. 1991). $D f(3 R) r o^{80 b}$ deletes the Toll locus. $g d^{8}$ is a null allele of gd. For general markers, see Lindsley and Zimm (1992).

\section{Labeling and immunoprecipitation of Dorsal}

Ovaries were dissected from Ore- $\mathrm{R}$ females (3-6 days old) in phosphate-free labeling medium (LM) consisting of balanced salt solution (BSS) (Ashburner 1989) supplemented with Grace's amino acids and vitamins (Sigma). One hundred pairs of ovaries were placed into $0.5 \mathrm{ml}$ of fresh LM to which $1 \mathrm{mCi}$ of $\left[{ }^{32} \mathrm{P}\right]$ orthophosphate was added. Labeling was carried out at room temperature for $3 \mathrm{hr}$ with gentle rocking. Approximately 50\%-70\% of the total counts were incorporated in the ovaries after this incubation. The labeled ovaries were homogenized on ice in extraction buffer (EB): $20 \mathrm{~mm}$ HEPES, $150 \mathrm{~mm} \mathrm{NaCl}, 1 \mathrm{~mm}$ EDTA, $0.5 \%$ Triton X-100, $1 \mathrm{~mm}$ phenylmethylsulfonyl fluoride, $10 \mu \mathrm{M}$ leupeptin, $1 \mu \mathrm{M}$ pepstatin, $0.3 \mu \mathrm{M}$ aprotinin, and 50 $\mathrm{mm} \mathrm{NaF}, 2 \mathrm{~mm}$ sodium vanadate (pH 7.5). Embryos were labeled by directly injecting $\left(\sim 1 \mathrm{pl} /\right.$ embryo) $\left[{ }^{32} \mathrm{P}\right]$ orthophosphate (250 $\mu \mathrm{Ci} / 10 \mu \mathrm{l}$ of injection buffer) into cleavage stage. After $\sim 2 \mathrm{hr}$ (at cellular blastoderm), the entire contents of each embryo were extracted with a large bore needle as described previously (Gillespie et al. 1994).

Extracts from both ovary and embryo labelings were lysed in
EB on ice for $30 \mathrm{~min}$ and then centrifuged at $13 \mathrm{~K}$ for $30 \mathrm{~min}$ at $4^{\circ} \mathrm{C}$ to remove insoluble material. Immunoprecipitation was performed as described (Whalen et al. 1993).

\section{Phosphoamino acid analysis}

Immunoprecipitated-labeled proteins separated on acrylamide gels were transferred to Immobilon (Millipore). Labeled Dorsal was identified on the basis of its molecular weight. The appropriate portion of the membrane was cut and the bound protein was subjected to total hydrolysis in $5.7 \mathrm{~N} \mathrm{HCl}$ (Kamps 1991). Phosphoamino acid analysis of the hydrolysate was done by TLC as described previously (Boyle et al. 1991). Cold phosphoserine, phosphothreonine, and phosphotyrosine (Sigma) were cospotted with the hydrolysate in sufficient quantities to detect via ninhydrin staining.

Selection of potential phosphorylation sites within the RHR of Dorsal

We confined our alterations to six highly conserved serines within the RHR of Dorsal (see Fig. 3). With the exception of S103, the conservation is not limited to the particular serine residues but extends to surrounding residues as well. Only two (S79 and S312) are recognized as consensus sites by the GCG sequence analysis program for identifying kinase recognition sites. S312 is a cAMP-dependent PKA site, and S79 matches a CKII phosphorylation site. To further rule out threonine and tyrosine phosphorylation, we made mutations in two conserved threonine residues (T85A, a Cdc2 site, and T290V, a protein kinase C site) and one conserved tyrosine residue (Y324F, a PI-3 kinase site) that were good matches to kinase-recognition targets.

\section{Construction of mutant transgenes and dorsal minigene}

A wild-type dorsal minigene was assembled with the dorsal cDNA fused to $\sim 4.5 \mathrm{~kb}$ of $5^{\prime}$-genomic DNA from the dorsal locus in pBSIIKS $^{+}$and pBSIIKS $^{-}$(Stratagene). Site-directed mutagenesis was carried out with these plasmids as templates and the appropriate primer (Kunkel 1985, 1995). The mutageneses were confirmed by dideoxy sequencing; in each case, the entire RHR was sequenced to confirm the presence of the mutation and rule out extraneous mutation events. The complete mutant minigenes were transferred to a CaSpeR P-element transformation vector with a modified polylinker via $K p n I$ and NotI.

\section{P-element mediated transformation and transgene analysis}

Transformation was carried out as described previously (Spradling 1986) with $w^{1118}$ flies and pTurbo as a transposase source. The endogenous regulatory region of the dorsal locus (see dorsal minigene above) confers line-independent wild-type levels of expression on dorsal cDNA as measured by rescue and protein levels from several independent lines (see Table 1 footnote). Transformant lines for each construct were isolated and crossed into the various backgrounds described to assess their in vivo effects on Dorsal function.

\section{Cuticle preparations and antibody staining}

Terminally differentiated embryos were collected, dechorionated in $50 \%$ bleach, devitellinized in methanol, fixed in acetic acid/glycerol (4:1), mounted in Hoyer's/lactic acid (1:1), and cleared overnight at $65^{\circ} \mathrm{C}$ as described (Wieschaus and Nüsslein-Volhard 1986). 
Embryo collections $(0-3 \mathrm{hr})$ were fixed and stained with pooled anti-Dorsal monoclonal antibodies, 7A4-39 and 7F12-15 (Whalen et al. 1993) as described (Ashburner 1989). To exactly compare nuclear import levels between wild-type, dorsal-null, and particular S-A mutant proteins, anti-Dorsal antibody stainings were done in parallel. The stainings were stopped when the wild-type embryos had developed sufficiently to visualize the Dorsal nuclear gradient well. In all cases, the parallel dorsalnull staining still showed little or no color. Representative embryos from each staining were chosen for all figures.

\section{Dorsal phosphoform analysis and Cactus Western blots}

Minigel PAGE was performed with a higher ratio of acrylamide to bisacrylamide (120:1, low-bis) to facilitate the separation of Dorsal phosphoforms (Whalen et al. 1993; Gillespie et al. 1994). Approximately thirty 0-3 hr embryos or one pair of ovaries were used per lane. The dephosphorylated Dorsal was prepared by incubating wild-type extracts in EB without phosphatase inhibitors at $30^{\circ} \mathrm{C}$ for $60 \mathrm{~min}$ as described (Gillespie et al. 1994).

\section{Acknowledgments}

We thank Marcia Belvin, Girish Deshpande, Kirsteen Munn, and Jerry Yin for their helpful discussions and comments on the manuscript, and Millie M. Georgiadis for help with questions concerning Dorsal structure. We thank Le Nguyen for fly food. This work was supported by grants from the National Institutes of Health and the Horace W. Goldsmith Foundation.

The publication costs of this article were defrayed in part by payment of page charges. This article must therefore be hereby marked 'advertisement' in accordance with 18 USC section 1734 solely to indicate this fact.

\section{References}

Anderson, K.V. and C. Nüsslein-Volhard. 1984. Genetic analysis of dorsal-ventral embryonic pattern in Drosophila. In Pattern formation (ed. G.M. Molacinski and S.V. Bryant), pp. 269-289. MacMillan, New York, NY.

Ashburner, M. 1989. Drosophila: A laboratory manual. Cold Spring Harbor Laboratory Press, Cold Spring Harbor, NY.

Baeuerle, P.A. and D. Baltimore. 1998. Activation of DNA-binding activity in an apparently cytoplasmic precursor of NF-кB transcription factor. Cell 53: 211-217.

Belvin, M.P., Y. Jin, and K.V. Anderson. 1995. Cactus protein degradation mediates Drosophila dorsal-ventral signaling. Genes \& Dev. 9: 783-793.

Bergmann, A., D. Stein, R. Geisler, S. Hagenmaier, B. Schmid, N. Fernandez, B. Schnell, and C. Nüsslein-Volhard. 1996. A gradient of cytoplasmic Cactus degradation establishes the nuclear localization gradient of the dorsal morphogen in Drosophila. Mech. Dev. 60: 109-123.

Boyle, W.J., P.v.d. Geer, and T. Hunter. 1991. Phosphopeptide mapping and phosphoamino acid analysis by two-dimensional separation on thin-layer cellulose plates. Methods Enzymol. 201: 110-149.

Briggs, L.J., D. Stein, J. Goltz, V.C. Corrigan, A. Efthymiadis, S. Hubner, and D.A. Jans. 1998. The cAMP-dependent protein kinase site $\left(\mathrm{Ser}^{312}\right)$ enhances Dorsal nuclear import through facilitating nuclear localization sequence/importin interaction. J. Biol. Chem. 273: 22745-22752.

Brown, K., S. Gerstberger, L. Carlson, G. Franzoso, and U. Siebenlist. 1995. Control of IкB- $\alpha$ proteolysis by site-spe- cific, signal induced phosphorylation. Science 267: 14851488.

Bushdid, P.B., D.M. Brantley, F.E. Yull, G.L. Blaeuer, L.H. Hoffman, L. Niswander, and L.D. Kerr. 1998. Inhibition of NF-кB activity results in disruption of the apical ectodermal ridge and abberant limb morphogenesis. Nature 392: 615-618.

Cao, Z., W.J. Henzel, and X. Gao. 1996. IRAK: A kinase associated with the interleukin-1 receptor. Science 271:11281131.

DiDonato, J.A., M. Hayakawa, D.M. Rothwarf, E. Zandi, and M. Karin. 1997. A cytokine-responsive IкB kinase that activates the transcription factor NF-кB. Nature 388: 548-554.

Drier, E.A. and R. Steward. 1997. The dorsoventral signal transduction pathway and the Rel-like transcriptions factors in Drosophila. Semin. Cancer Biol. 8: 83-92.

Dubnicoff, T., S.A. Valentine, G. Chen, J.A. Lengyel, Z.E. Paroush, and A.J. Courey. 1997. Conversion of Dorsal from an activator to a repressor by the global corepressor Groucho. Genes \& Dev. 11: 2952-2957.

Edwards, D.N., P. Towb, and S.A. Wasserman. 1997. An activity-dependent network of interactions links the Rel protein Dorsal with its cytoplasmic regulators. Development 124: 3855-3864.

Erdelyi, M. and J. Szabad. 1989. Isolation and characterization of dominant female sterile mutations of Drosophila melanogaster. I. Mutations on the third chromosome. Genetics 122: $111-127$.

Geisler, R., A. Bergmann, Y. Hiromi, and C. Nüsslein-Volhard. 1992. cactus, a gene involved in dorsoventral pattern formation of Drosophila, is related to the IкB family of vertebrates. Cell 71: 613-621.

Ghosh, G., G.V. Duyne, S. Ghosh, and P.B. Sigler. 1995. Structure of NF- $\mathrm{BB}$ p50 homodimer bound to a $к \mathrm{~B}$ site. Nature 373: 303-310.

Gillespie, S.K.H. and S. Wasserman. 1994. dorsal, a Drosophila rel-like protein, is phosphorylated upon activation of the transmembrane protein Toll. Mol. Cell. Biol. 14: 3559-3568.

Govind, S., L. Brennan, and R. Steward. 1993. Homeostatic balance between dorsal and cactus proteins in the Drosophila embryo. Development 117: 135-148.

Govind, S., E. Drier, L.H. Huang, and R. Steward. 1996. Regulated nuclear import of the Drosophila Rel protein Dorsal: Structure-function analysis. Mol. Cell. Biol. 16: 1103-1114.

Hashimoto, C., K.L. Hudson, and K.V. Anderson. 1988. The Toll gene of Drosophila, required for dorsal-ventral embryonic polarity, appears to encode a transmembrane protein. Cell 52: 269-279

Hecht, P.M. and K.V. Anderson. 1993. Genetic characterization of tube and pelle, genes required for signaling between Toll and dorsal in the specification of the dorsal-ventral pattern of the Drosophila embryo. Genetics 135: 405-417.

Ip, Y.T., R. Kraut, M. Levine, and C.A. Rushlow. 1991. The dorsal morphogen is a sequence-specific DNA-binding protein that interacts with a long-range repression element in Drosophila. Cell 64: 439-446.

Isoda, K., S. Roth, and C. Nüsslein-Volhard. 1992. The functional domains of the Drosophila morphogen dorsal: Evidence from the analysis of mutants. Genes \& Dev. 6: 619630.

Jiang, J., D. Kosman, Y.T. Ip, and M. Levine. 1991. The dorsal morphogen gradient regulates the mesoderm determinant twist in early Drosophila embryos. Genes \& Dev. 5: 18811891.

Jiang, J., H. Cai, Q. Zhou, and M. Levine. 1993. Conversion of a dorsal-dependent silencer into an enhancer: Evidence for dorsal coreppressors. EMBO J. 12: 3201-3209. 
Kanegae, Y., A.T. Tavares, J.C.I. Belmonte, and I. Verma. 1998. Role of Rel/NF-кB transcription factors during the outgrowth of the vertebrate limb. Nature 392: 611-615.

Kidd, S. 1992. Characterization of the Drosophila cactus locus and analysis of interaction between cactus and dorsal proteins. Cell 71: 623-635.

Kirov, N., L. Zhelnin, J. Shah, and C. Rushlow. 1993. Conversion of a silencer into an enhancer: Evidence for a co-repressor in dorsal-mediated repression in Drosophila. EMBO $J$. 12: 3193-3199.

Kunkel, T.A. 1985. Rapid and efficient site-specific mutagenesis without phenotypic selection. Proc. Natl. Acad. Sci. 82: 488-492.

. 1995. Oligonucleotide-directed mutagenesis without phenotypic selection. In Current protocols in molecular biology (ed. F.M. Ausubel, R. Brent, R.E. Kingston, D.D. Moore, J.G. Seidman, J.A. Smith, and K. Struhl). John Wiley, New York, NY.

Letsou, A., S. Alexander, K. Orth, and S.A. Wasserman. 1991. Genetic and molecular characterization of tube, a Drosophila gene maternally required for embryonic dorsoventral polarity. Proc. Natl. Acad. Sci. 88: 810-814.

Li, C.C., M. Korner, D.K. Ferris, E. Chen, R.M. Dai, and D.L. Longo. 1994. NF-кB/Rel family members are physically associated phosphoproteins. Biochem. J. 303: 499-506.

Lindsley, D.L. and G.G. Zimm. 1992. The genome of Drosophila melanogaster. Academic Press, San Diego, CA.

Medzhitov, R., P. Preston-Hurlburt, and C.A. Janeway. 1997. A human homologue of the Drosophila Toll protein signals activation of adaptive immunity. Nature 388: 394-397.

Mercurio, F., H. Zhu, B.W. Murray, A. Shevchenko, B.L. Bennet, J.W. Li, D.B. Young, M. Barbosa, M. Mann, A. Manning, and A. Rao. 1997. IKK-1 and IKK-2: Cytokine-activated IкB kinases essential for NF-кB activation. Science 278: 860-866.

Morisato, D. and K.V. Anderson. 1995. Signaling pathways that establish the dorsal-ventral pattern of the Drosophila embyo. Annu. Rev. Genet. 29: 371-399.

Muller, C.W., F.A. Rey, M. Sodeoda, G.L. Verdine, and S.C. Harrison. 1995. Structure of the NF-кB p50 homodimer bound to DNA. Nature 373: 311-317.

Naumann, M. and C. Scheidereit. 1994. Activation of NF-кB in vivo is regulated by multiple phosphorylations. EMBO $\mathrm{I}$. 13: 4597-4607.

Norris, J.L. and J.L. Manley. 1992. Selective nuclear transport of the Drosophila morphogen dorsal can be established by a signaling pathway involving the transmembrane protein Toll and protein kinase A. Genes \& Dev. 6: 1654-1667.

Ray, R.P., K. Arora, C. Nüsslein-Volhard, and W. Gelbart. 1991. The control of cell fate along the dorsal-ventral axis of the Drosophila embryo. Development 113: 35-54.

Reach, M., R.L. Galindo, P. Towb, J.L. Allen, M. Karin, and S. Wasserman. 1996. A gradient of Cactus protein degradation establishes dorsoventral polarity in the Drosophila embryo. Dev. Biol. 180: 353-364.

Roth, S., D. Stein, and C. Nüsslein-Volhard. 1989. A gradient of nuclear localization of the dorsal protein determines dorsoventral pattern in the Drosophila embryo. Cell 59: 11891202.

Roth, S., Y. Hiromi, D. Godt, and C. Nüsslein-Volhard. 1991. cactus, a maternal gene required for proper formation for the dorsoventral morphogen gradient in Drosophila embryos. Development 112: 371-388.

Rusch, J. and M. Levine. 1994. Regulation of the dorsal morphogen by the Toll and torso signaling pathways: A receptor tyrosine kinase selectively masks transcriptional repression. Genes \& Dev. 8: 1247-1257.
Rushlow, C.A., K. Han, J.L. Manley, and M. Levine. 1989. The graded distribution of the dorsal morphogen is initiated by selective nuclear transport in Drosophila. Cell 59: 11651177.

Schneider, D.S., K.L. Hudson, T.Y Lin, and K.V. Anderson. 1991. Dominant and recessive mutations define functional domains of Toll, a transmembrane protein required for dorsal ventral polarity in the Drosophila embryo. Genes \& Dev. 5: 797-807.

Schüpbach, T. and E. Wieschaus. 1989. Female sterile mutations on the second chromosome of Drosophila melanogaster. I. maternal effect mutations. Genetics 121: 101-117.

Sen, R. and D. Baltimore. 1986. Inducibility of $\kappa$ immunoglobulin enhancer-binding protein NF-кB by a posttranslational mechanism. Cell 47: 921-928.

Shelton, C.A. and S.A. Wasserman. 1993. pelle encodes a protein kinase required to establish dorsoventral polarity in the Drosophila embryo. Cell 72: 515-525.

Siebenlist, U., G. Franzoso, and K. Brown. 1994. Structure, regulation and function of NF-кB. Annu. Rev. Cell Biol. 10: 405455.

Spradling, A.C. 1986. P element mediated transformation. In Drosophila: A practical approach (ed. D. Roberts), pp. 175197. IRL Press, Oxford, UK

Steward, R. 1989. Relocalization of the dorsal protein from the cytoplasm to the nucleus correlates with its function. Cell 59: $1179-1188$.

Steward, R. and C. Nüsslein-Volhard. 1986. The genetics of the dorsal-bicaudal-D region of Drosophila melanogaster. Genetics 113: 665-678.

Verma, I.M., J.K. Stevenson, E.M. Schwarz, D.V. Antwerp, and S. Miyamoto. 1995. Rel/NF-кB/ІкB family: Intimate tales of association and dissociation. Genes \& Dev. 9: 2723-2735.

Whalen, A.M. and R. Steward. 1993. Dissociation of the DorsalCactus complex and phosphorylatin of the Dorsal protein correlate with the nuclear localization of Dorsal. J. Cell Biol. 123: $523-534$

Whiteside, S.T. and A. Isreal. 1997. ІкB proteins: Structure, function, and regulation. Semin. Cancer Biol. 8: 75-82.

Wieschaus, E. and C. Nüsslein-Volhard. 1986. Looking at embryos. IRL Press, Oxford, Washington, DC.

Woronicz, J.D., X. Gao, Z. Cao, M. Rothe, and D.V. Goeddel. 1997. ІкB kinase- $\beta$ : NF- $\kappa B$ activation and complex formation with IкB kinase- $\alpha$ and NIK. Science 278: 866-869.

Yang, J. and R. Steward. 1997. A multimeric complex and the nuclear targetting of the Drosophila Rel protein, Dorsal. Proc. Natl. Acad. Sci. 94: 14524-14529.

Zandi, E., D.M. Rothwarf, M. Delhase, M. Hayakawa, and M. Karin. 1997. The IкB kinase complex (IKK) contains two kinase subunits, IKK $\alpha$ and IKK $\beta$, necessary for IкB phosphorylation and NF- $\kappa$ B activation. Cell 91: 243-252.

Zhong, H., R.E. Voll, and S. Ghosh. 1998. Phosphorylation of NF- $\kappa$ B p 65 by PKA stimulates transcriptional activity by promoting a novel bivalent interaction with the coactivator CBP/p300. Mol. Cell 1: 661-671. 


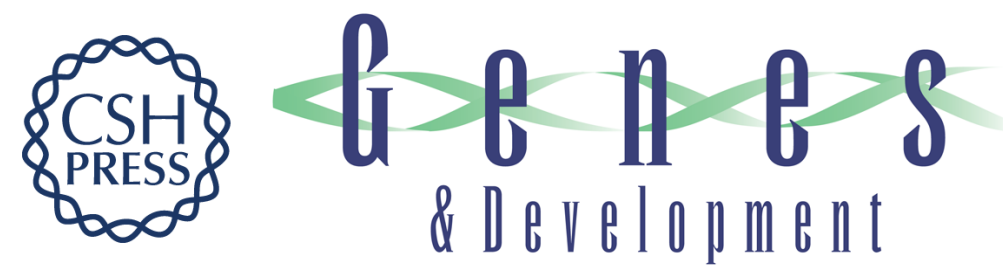

\section{Nuclear import of the Drosophila Rel protein Dorsal is regulated by phosphorylation}

Eric A. Drier, Leslie H. Huang and Ruth Steward

Genes Dev. 1999, 13:

References This article cites 54 articles, 27 of which can be accessed free at:

http://genesdev.cshlp.org/content/13/5/556.full.html\#ref-list-1

License

Email Alerting Receive free email alerts when new articles cite this article - sign up in the box at the top Service right corner of the article or click here.

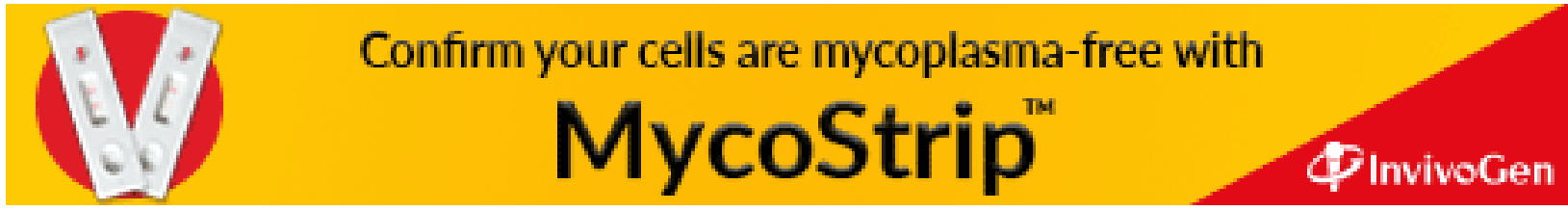

